ISSN: 2521-5051 (Print)

ISSN: 2521-506X (online)

CODEN : ASMCCQ

\title{
A STUDY ON SOME FUNDAMENTAL CHARACTERISTICS OF SEQUENCES OF SOFT REAL NUMBERS
}

\author{
Arif Mehmood Khattak ${ }^{1}$, Saleem Abdullah ${ }^{2}$, Zaheer Anjum Khattak ${ }^{3}$
}

${ }^{1}$ Department of Mathematics and Statistics Riphah International University, Sector I-14 Islamabad (Pakistan)

${ }^{2}$ Abdul Wali Khan University Mardan Department of Mathematics (Pakistan)

${ }^{3}$ Bannu University of Science and Technology Department of Mathematics (Pakistan)

This is an open access article distributed under the Creative Commons Attribution License, which permits unrestricted use, distribution, and reproduction in any medium, provided the original work is properly cited

\section{ARTICLE DETAILS \\ ABSTRACT}

\section{Article History:}

Received 12 November 2017 Accepted 12 December 2017 Available online 1 January 2018

The main aim of this article is to discuss soft limit, convergent sequences, Soft boundedness, and Soft convergent Divergent criterion. Soft monotone sequences, Soft squeezness, Soft Cauchy Sequence, Soft Cauchy boundedness and Soft Cauchy first and second theorem. Uniqueness of soft limit of soft convergent sequences and related results, Soft monotone sequences and soft monotone criterion.

\section{KEYWORDS}

Soft sequence, soft limit, soft convergence, soft monotone, soft sub sequences, Soft boundedness and Soft squeeze result. Soft Cauchy sequence, Soft Cauchy results

\section{INTRODUCTION}

Following the seminal work of a study on fuzzy set theory, building of mathematical theory and their applications in handling the problems under uncertain atmosphere has been getting momentum day by day [1]. Supposing some difficulties in the parameterization process in Fuzzy set theory in 1999 introduced the idea of soft set as a parameterized family of sets where parameter set takes values from an arbitrary set [2]. He also showed the applications of soft set in smoothness of functions, probability theory, measure theory, and game theory etc. After that P.K.Maji et al defined some operations on soft sets based on introduced soft topologies [3-5].

Aktas and Cagman soft group; Nazmul and Samanta soft topological group. Recently Das and Samanta introduced the idea of soft metric and soft normed linear space and also discussed soft real numbers in equalities [69]. Das and Samanta also introduced the concept of soft real numbers [1013]. Using this concept, they studied some basic properties of soft real numbers. However, in their study on functions they have considered functions over crisp sets and have used extension principle for getting images of soft sets. In 2016, a studied some basic operations like supremum, infimum, accountability in soft setting [11]. made discussion on soft functions and discussed some fundamental results namely, Bolzano result, Intermediate result. He also beautifully extended his approach to the notion of soft differentiation. With base of soft derivative, he touched important result which is known as the back bone of soft real analysis that result is Rolle's Theorem. Soft Lagrange's theorem is also discussed in soft setting.

No one tried their hands on Soft Sequences theorems at detail. In this present paper soft sequences result are discussed. Soft convergent sequences are defined. Uniqueness Theorem is proved in soft setting. Soft monotone sequences, soft monotonically increasing, soft monotonically decreasing results are discussed, and their convergence and divergence are discussed. Squeeze Theorem which plays important role in soft real analysis is discussed in soft setting. Soft Boundedness of soft sequences are studied, and some results related to soft boundedness are also reflected here. Soft Sub sequences and their convergent behaviors are also discussed. It is showed that every soft sub sequence of soft convergent is soft convergent and the soft limit of the soft sub sequence is the same as that of the soft sequence. Since the Soft Monotone convergence results are extra-ordinary useful and important, but it has the significant draw-back that it applies only to soft sequences that are monotone. It is important to us to have a condition implying the convergence of a soft sequence that does not require us to know the value of the soft limit in advance, and it is not restricted to soft monotone sequence. This problem is finished out by Cauchy by providing a general principle of soft convergence.

The soft fundamental sequence plays significant part in soft real analysis The importance of this concept lies in the main theorem, which asserts that a soft sequence of soft real numbers is soft convergent if and only if it is a soft Cauchy Sequence. This give us an interested technique of proving a soft sequence soft convergence without knowing the soft limit of the soft sequence. Soft Sub sequences of soft sequences plays important rule in soft real analysis. Sometimes on the basis of soft sub sequences we can get knowledge about the main sequence. Soft sub sequences will definitely possess the characteristics of the sequence from which the sub sequence is generated. Sub sequences are produced for a definite purpose. For, Instance, soft sub sequences are often useful in establishing the soft convergence or the soft divergence of the given soft sequence. Bolzano theorem is more informative about sub sequences. We know that a soft convergent soft sequence is bounded. But the converse is not true. If a soft sequence is un-bounded it may or may not have a convergent soft sub sequence. So a natural question arises if a soft sequence is bounded, can we always extract a soft sub sequence that is soft convergent. The answer is in yes. This yes is beautifully defended by a famous Bolzao- Weierstrass Theorem.

\section{PRELIMINARIES}

Definition1: [10]. Let $X$ be an initial universe of discourse and $\mathrm{E}$ be a set of parameters. Let $\mathrm{P}(\mathrm{X})$ denotes the power set of $X$ and A be a nonempty sub-set of E. A pair $(\boldsymbol{F}, \boldsymbol{A})$ is called a soft set over $\mathrm{U}$, where $\mathrm{F}$ is a mapping given by $F: A \rightarrow P(X)$

In other words, a set over $X$ is a parameterized family of sub set of universes of discourse $X$. For $e \in A, F(e)$ may be considered as the set of e-approximate elements of the soft set $(F, A)$ and ife $\notin A$ then $F(e)=\phi$, that is $F_{A=}\{F(e): e \in A \subseteq E, F: A \rightarrow P(X)\}$ the family of all these soft sets over $\mathrm{X}$ denoted bySS $(X)_{A}$.

Definition2: [10]. Let $F_{A} G_{B} \in S S(X)_{E}$ then $F_{A}$, is a soft subset of $G_{B}$ denoted by $F_{A} \subseteq G_{B}$, if

1. $\quad A \subseteq B$ and

2. $F(e) \subseteq G(e), \forall \in A$

In this case $F_{A}$ is said to be a soft subset of $G_{B}$ and $G_{B}$ is said to be a soft super set $F_{A}, G_{B} \supseteq F_{A}$. 
Definition3: [10]. Two soft subsets $F_{A}$ and $G_{B}$ over a common universe of discourse set $\mathrm{X}$ are said to be equal if $F_{A}$ is a soft subset of $G_{B}$ and $G_{B}$ is a soft subset of $F_{A}$.

Definition4: [10].The complement of soft $\operatorname{subset}(F, A)$ ddenoted by $(F, A)^{C}$ is defined by $(F, A)^{C}=\left(F^{C}, A\right) F^{C} \rightarrow P(X)$ is a mapping given by $F^{C}(e)=U-F(e) \forall e \in A$ and $F^{C}$ is called the soft complement function of $F$. Clearly $\left(F^{C}\right)^{C}$ is the same as $F$ and $\left((F, A)^{C}\right)^{C}=(F, A)$.

Definition5: [10].The difference between two soft subset $(G, E)$ and $(G, E)$ over common of universe discourse $X$ denoted by $(F, E)-(G, E)$ is the soft $\operatorname{set}(H, E)$ such that $H(e)=F(e) \backslash G(e)$ for alle $\in E$.

Definition6: [10]. The union of two soft $\operatorname{sets}(F, A)$ and $(\mathrm{G}, B)$ over the common universe of discourse $\mathrm{X}$ is the $\operatorname{soft} \operatorname{set}(H, C)$, where, $C=$ $A U B \forall e \in C$

$H(e)=\left\{\begin{array}{cc}F(e) & \text { if } e \in A-B \\ G(e) & \text { if } e \in(B-A) \\ F(e), & \text { if } e \in A \cap B\end{array}\right\}$

Written as $(F, A) \cup(G, B)=(H, C)$

Definition7: [10]. The intersection (H.C) of two soft sets $(F, A)$ and $(G, B)$ over common universe $\mathrm{X}$, denoted $(F, A) \bar{\cap}(\mathrm{G}, \mathrm{B})$ is defined as $C=$ $A \cap B$ and $H(e)=F(e) \cap G(e), \forall e \in C$.

Definition8: [13]. Let $\mathrm{R}$ be the set of real numbers, $\mathrm{P}(\mathrm{R})$ be the collection of all nonempty bounded subsets of $\mathrm{R}$ and $\mathrm{A}$ be a set of parameters. Then a mapping F: $A \rightarrow P(R)$ is called a soft real set. It is denoted by $(F, A)$.

If in particular $(F, A)$ is a singleton soft set, then identifying $(F, A)$ with the corresponding soft element, it will be called a soft real number.

We use the notation $\sim x, \tilde{y}, \tilde{z}$ to denote soft real numbers whereas $\overline{\boldsymbol{x}}, \overline{\boldsymbol{y}}, \overline{\boldsymbol{z}}$ will denote a particular type of soft real numbers such that $\overline{\boldsymbol{x}}(\lambda)=$ $\boldsymbol{x}$ for all $\lambda \in \mathrm{A}$ etc. Note that, in general, $\tilde{x}$ is not related to $\mathrm{x}$.

Definition9: [8]. For two soft real numbers $\tilde{r}, \tilde{s}$ we define

(1) $\tilde{r} \widetilde{\geq} \tilde{s}$ if $\tilde{r}(\lambda) \widetilde{\Sigma} \tilde{s}(\lambda)$, for all $\lambda \in \mathrm{A}$.

(2) $\tilde{r} \widetilde{\widetilde{s}} \tilde{s}$ if $\tilde{r}(\lambda) \widetilde{\widetilde{s}} \tilde{s}(\lambda)$, for all $\lambda \in \mathrm{A}$.

(3) $\tilde{r} \widetilde{\widetilde{s}} \tilde{s}$ if $\tilde{r}(\lambda) \widetilde{\widetilde{s}} \tilde{s}(\lambda)$, for all $\lambda \in \mathrm{A}$.

(4) $\tilde{r} \widetilde{<} \tilde{s}$ if $\tilde{r}(\lambda) \widetilde{\gtrless} \tilde{s}(\lambda)$, for all $\lambda \in \mathrm{A}$.

Theorem 1 [8] for any soft set $(F, A), \operatorname{SS}(\mathrm{SE}((\mathrm{F}, \mathrm{A})))=(\mathrm{F}, \mathrm{A})$ (where $\mathrm{SE}(\mathrm{F}$, $A)=\{\sim x: \sim x(\lambda) \in F(\lambda), \forall \lambda \in A\}$ and SS (B) $(\lambda)=\{\sim x(\lambda): \sim x \in B\}$, Where B is a set of soft real numbers).

Definition10: [11]. A real soft set $(F, A)$ is said to be bounded from above if there exists a soft real number ${ }^{\sim} r$ such that ${ }^{\sim} x^{\sim} \leq^{\sim} r, \forall^{\sim} x^{\sim} \in(F, A)$.

Definition11: [12]. A real soft set $(F, A)$ is said to be bounded from below if there exists a soft real number ${ }^{\sim} r$ such that ${ }^{\sim} x^{\sim}{ }^{\sim} r, \forall^{\sim} x^{\sim} \in(F, A)$.

\section{LIMIT OF A SOFT SEQUENCE SOFT CONVERGENT SEQUENCE}

Definition12: If the sequence $\left\langle a_{n}^{\sim}>\right.$ of soft real numbers has the limit $\tilde{l^{2}}$ we

say that the sequence $<\tilde{\tilde{a}}_{n}>$ is convergent to soft limit $\tilde{\tilde{l}}$ if $<\tilde{\tilde{a}}_{n}>$ does not have soft limit we say that $<\tilde{\tilde{a}}_{n}>$ is divergent.

Definition13: Let $\left\langle a_{n}^{\sim}\right\rangle$ be a sequence of soft real numbers we say that $a_{n}^{\sim}$ approaches the soft limit $\sim$ (as $\mathrm{n}$ tends to infinity) if for every soft real number $\epsilon^{\sim \sim}>0^{-}$there is soft positive integer $m^{\sim \sim}\left(\epsilon^{\sim \sim}\right)$ depending upon $\epsilon^{\sim \sim}$ such

that $\left|\tilde{\tilde{a}}_{n}-\tilde{\tilde{l}}\right| \widetilde{<} \widetilde{\widetilde{\epsilon}}$, for all $n \widetilde{\widetilde{z}} m(\widetilde{\widetilde{\epsilon}})$ if $\tilde{a}_{n}$ approaches the soft limit $\tilde{l}$ we write $\lim _{n \rightarrow \infty} \tilde{\tilde{a}}_{n}=\tilde{l}$ or $\quad \tilde{\tilde{a}}_{n} \rightarrow \tilde{l}$ as $n \rightarrow \infty$

$n \rightarrow \infty$
Remark- (i) the expression.

$\left|\tilde{\tilde{a}}_{n}-\tilde{\tilde{l}}\right| \widetilde{\widetilde{\varepsilon}} \widetilde{\widetilde{\epsilon}}$

$-\widetilde{\tilde{\epsilon}} \widetilde{\tilde{a}_{n}}-\tilde{l} \widetilde{<} \widetilde{\epsilon}$

$\widetilde{\epsilon}-\widetilde{\epsilon} \widetilde{<} \tilde{\tilde{a}}_{n} \widetilde{<} \tilde{\tilde{l}}+\widetilde{\widetilde{\epsilon}}$

$\tilde{\tilde{a}}_{n} \widetilde{\widetilde{\epsilon}}(\tilde{\tilde{l}}-\tilde{\widetilde{\epsilon}}, \tilde{\tilde{l}}+\widetilde{\widetilde{\epsilon}})$

Thus if $\tilde{\tilde{l}}$ is the soft limit of the soft sequence $<\tilde{\tilde{a}}_{n}>$ then for every soft real number $\widetilde{\tilde{\epsilon}}>\overline{0}$ there exists a soft positive integer $\widetilde{\widetilde{m}}(\widetilde{\tilde{\epsilon}})$ depending upon $\widetilde{\widetilde{\epsilon}}$ such that from and after the $\mathrm{m}^{\text {th }}$ term $\tilde{\tilde{a}}_{m}$ of the sequence all terms $\tilde{\tilde{a}}_{n}$ belongs to the open interval

$(\tilde{\tilde{l}}-\widetilde{\widetilde{\epsilon}} \tilde{\tilde{l}}+\widetilde{\widetilde{\epsilon}})$, that is, if $\tilde{\tilde{l}}>\tilde{s}$ the soft limit then the open interval $(\tilde{\tilde{l}}-\widetilde{\widetilde{\epsilon}}, \tilde{l}+$

$\widetilde{\widetilde{\epsilon}}$ ) contains $\tilde{\tilde{a}}_{n}$ infinite numbers o terms (soft points) of the soft sequence then $\tilde{l}$ is not a soft limit points of the sequence.

2) if limit $\tilde{\tilde{a}}_{n}=\tilde{l}$ then for $n \rightarrow \infty$ given $\widetilde{\in} \widetilde{\bar{S}} \overline{0}$, the value of $m$ should come out finite and positive. if $m$ is not determined, then the soft sequence < $\tilde{\tilde{a}}_{n}>$ does not have a soft limit.

A sequence $<\tilde{x}_{n}>$ of soft elements $\tilde{\tilde{x}}$ is said to be soft convergent and converges to soft elements $\tilde{\tilde{x}}$ if $\left|\tilde{\tilde{x}}_{n}-\tilde{\tilde{x}}\right| \widetilde{<} \widetilde{\epsilon}$, for all $n \widetilde{\mathbb{x}} m(\widetilde{\widetilde{\epsilon}})$ if $\tilde{\tilde{x}}_{n}$ converges to $\tilde{\tilde{x}}$, we write $\tilde{\tilde{x}}_{n} \rightarrow \tilde{\tilde{x}}$ as $n \rightarrow \infty$

$\left|\tilde{\tilde{x}}_{n}-\tilde{\tilde{x}}\right| \widetilde{\widetilde{\varepsilon}} \widetilde{\epsilon}$, for all $n \widetilde{\underline{\Sigma}} m(\widetilde{\widetilde{\epsilon}})$.

Definition14: If the sequence $\left\langle\tilde{a}_{n}^{\sim}\right\rangle$ of soft real numbers has the limit $l^{\sim}$ we say that the sequence $\left\langle a_{n}^{\sim}{ }_{n}>\right.$ is convergent and converges to soft limit liff $\left\langle a_{n}^{\sim}\right\rangle$ does not have soft limit we say that $\left\langle a_{n}^{\sim}\right\rangle$ is divergent.

Theorem 2. If $<\tilde{\tilde{a}}_{n}>$ is soft sequence of non-negative soft numbers and if $\lim _{n \rightarrow \infty} \tilde{\tilde{a}}_{n}=\tilde{\tilde{l}}$ then $\tilde{\tilde{l}} \widetilde{\geq}$.

Proof: We prove this by contradiction let $\tilde{l} \widetilde{<} \overline{0}$. Then for $\widetilde{\in}=-\tilde{l} / 2$, there exist such that soft real number $\widetilde{\widetilde{m}} \widetilde{\in} N$ such that $\left|\tilde{a}_{n}-\tilde{\tilde{l}}\right| \widetilde{<}-\tilde{\tilde{l}} / 2, n \widetilde{\geq} m$ In particular

$\left|\tilde{\tilde{a}}_{m}-\tilde{\tilde{l}}\right| \widetilde{<}-\tilde{l} / 2$

$\tilde{\tilde{a}}_{m}-\tilde{\tilde{l}} \widetilde{<}-\tilde{\tilde{l}} / 2$

$\tilde{\tilde{a}}_{m} \approx \tilde{<} \tilde{l}-\tilde{\tilde{l}} / 2$

$\tilde{\tilde{a}}_{m} \approx 2 \tilde{\tilde{l}}-\tilde{\tilde{l}} / 2$

$\tilde{\tilde{a}}_{m} \approx \tilde{<} \tilde{l} / 2$

$\tilde{\tilde{a}}_{m} \widetilde{<} \overline{0}$ But by hypothesis $\tilde{\tilde{a}}_{m} \widetilde{\geq} \overline{0}$. Hence this contradiction proves $\tilde{\tilde{l}} \widetilde{\geq} \overline{0}$.

Theorem 3. Let $\lim _{n \rightarrow \infty} \widetilde{\boldsymbol{a}}_{\boldsymbol{n}}=\tilde{\tilde{l}}, \lim _{n \rightarrow \infty} \widetilde{\widetilde{\boldsymbol{a}}}_{\boldsymbol{n}+\boldsymbol{p}}=\widetilde{\widetilde{\boldsymbol{l}}}, \boldsymbol{p} \widetilde{\tilde{\epsilon}} N$

Proof: since $<\tilde{\tilde{a}}_{n \rightarrow \infty}>$ converges to soft limit $\tilde{l}$, then for every $\widetilde{\in} \widetilde{S} \overline{0}$, there exists a positive integer $\mathrm{m}$ such that $\left|\tilde{a}_{n}-\tilde{\tilde{l}}\right| \widetilde{<} \widetilde{\in}$ implies $\bar{n} \widetilde{\Xi} \bar{m}$ implies $\left|\tilde{a}_{n}-\tilde{l}\right| \widetilde{\widetilde{\varepsilon}} \widetilde{\epsilon}$. So if we take $\overline{n_{0}}=n+p, p \widetilde{\widetilde{\epsilon}} N$. Then $n \widetilde{>} \overline{n_{0}}$ implies $\left|\tilde{\tilde{a}}_{n+p}-\tilde{l}\right| \widetilde{<} \widetilde{\tilde{\epsilon}}$. Hence $\lim _{n \rightarrow \infty} \tilde{\tilde{a}}_{n+p}=\tilde{\tilde{l}}$.

Theorem 4. Let $<\tilde{\tilde{a}}_{n}>$ be a soft convergent sequence of soft real numbers if

$\lim _{n \rightarrow \infty} \widetilde{\widetilde{a}}_{\boldsymbol{n}}=\tilde{\tilde{l}}$ and

$\lim _{n \rightarrow \infty} \widetilde{\widetilde{a}}_{n}=\widetilde{\boldsymbol{m}}$ Then $\tilde{\tilde{l}}=\widetilde{\boldsymbol{m}}$.

Proof: since $<\tilde{a}_{n}>$ converges to soft limit $\tilde{\tilde{l}}$ and $\widetilde{\widetilde{m}}$, then for every $\widetilde{\in} \widetilde{\bar{S}} \overline{0}$ there exists soft integer $\bar{r}$ and $\bar{s}$ such that $\bar{n} \widetilde{\Xi} \bar{r} \Rightarrow\left|\tilde{\tilde{a}}_{n}-\tilde{l}\right| \widetilde{\widetilde{<}} \widetilde{\widetilde{\epsilon}} / 2$ and $\bar{n} \widetilde{\widetilde{s}} \Rightarrow\left|\tilde{\tilde{a}}_{n}-\widetilde{\widetilde{m}}\right| \widetilde{<} \widetilde{\epsilon} / 2$

For $\widetilde{\widetilde{N}}=\max (\bar{r}, \bar{s})$

$n \widetilde{\Sigma} \widetilde{N} \Rightarrow\left|\tilde{\tilde{a}}_{n}-\tilde{\tilde{l}}\right| \widetilde{<} \widetilde{\epsilon} / 2$

$n \widetilde{\Xi} \widetilde{\widetilde{N}} \Rightarrow\left|\tilde{\tilde{a}}_{n}-\tilde{\widetilde{m}}\right| \widetilde{<} \widetilde{\epsilon} / 2$

$|\tilde{l}-\tilde{\widetilde{m}}|=\left|\tilde{\tilde{l}}-\tilde{\tilde{a}}_{n}+\tilde{\tilde{a}}_{n}-\tilde{\widetilde{m}}\right|$

$\widetilde{\widetilde{<}}\left|\tilde{\tilde{l}}-\tilde{\tilde{a}}_{n}\right|+\left|\tilde{\tilde{a}}_{n}-\tilde{\tilde{m}}\right|$

$\widetilde{<} \widetilde{\epsilon} / 2+\widetilde{\epsilon} / 2=\widetilde{\epsilon}$

Since $\widetilde{\in}$ was arbitrary we conclude that $|\tilde{l}-\widetilde{m}|=\overline{0}$

Or $\quad \tilde{l}-\tilde{m}=0$ or $\tilde{\tilde{l}}=\tilde{\bar{m}}$. If this theorem were not true, the definition of soft limit of a convergent soft sequence would have been meaningless.

Theorem 5. Let $<\tilde{a}_{n}>$ be a soft convergent sequence of soft real numbers and converges $\tilde{l}$ and moreover if $\tilde{\tilde{a}}_{n} \widetilde{\geq} \bar{a} \forall n \in N$ Then $\tilde{\tilde{l}} \widetilde{\mathrm{a}}$.

Proof: since $<\tilde{\tilde{a}}_{n}>$ converges to soft limit $\tilde{l}$ so according to definition $\lim _{n \rightarrow \infty} \tilde{a}_{n}=\tilde{l}$. We prove this result by contradiction let $\tilde{l} \widetilde{\widetilde{a}} \bar{a}$ this implies that $\tilde{l}-\bar{a} \widetilde{<} \overline{0}$ implies that $-\tilde{l}+\bar{a} \widetilde{>} \overline{0}$ implies that $\bar{a}-\tilde{l} \tilde{>} \widetilde{0}$. Since $\lim _{n \rightarrow \infty} \tilde{\tilde{a}}_{n}=\tilde{l}$

So then for every $\widetilde{\in} \widetilde{\bar{S}} \overline{0}$ there exists soft integer $m$ such that $\left|\tilde{\tilde{a}}_{n}-\tilde{\tilde{l}}\right|$ $\widetilde{\widetilde{\varepsilon}} \widetilde{\tilde{\epsilon}}$ for all $n \widetilde{\mathbb{Z}} m \ldots \ldots . . .(8)$. Since the choice of $\widetilde{\tilde{\epsilon}}$ is arbitrary so let us choose in particular $\widetilde{\in}=\bar{a}-\tilde{l}$ the clearly $\widetilde{\in} \widetilde{\bar{S}} \overline{0}$ as $\bar{a}-\tilde{l} \tilde{\bar{s}} \overline{0}$.so the above result (8) becomes $\left|\tilde{\tilde{a}}_{n}-\tilde{l}\right| \widetilde{\widetilde{\varepsilon}}=\bar{a}-\tilde{l}$, for all $n \widetilde{\widetilde{\geq}} m$ this implies $\left|\tilde{\tilde{a}}_{n}-\tilde{l}\right|$ $\widetilde{<} \bar{a}-\tilde{l}$ for all $n \widetilde{\widetilde{\Sigma}} m$ implies that $-(\bar{a}-\tilde{\tilde{l}}) \widetilde{\widetilde{\alpha}} \tilde{\tilde{a}}_{n}-\tilde{\tilde{l}} \widetilde{<} \bar{a}-\tilde{\tilde{l}}$ for all $n \widetilde{\geq} m$ implies that

$-\bar{a}+2 \tilde{\tilde{l}} \widetilde{\widetilde{\alpha}} \tilde{\tilde{a}}_{n} \widetilde{\widetilde{\alpha}} \bar{a}$ For all $n \widetilde{\widetilde{\Sigma}} m$. This implies that $\widetilde{\approx} \tilde{a}_{n} \widetilde{<} \bar{a}$ for 
all $n \widetilde{\geq} m$. Which is clearly a contradiction. Hence $\tilde{\tilde{l}} \widetilde{\underline{\geq}} \bar{a}$. This finishes the proof.

Theorem 6. Let $<\tilde{\tilde{a}}_{n}>$ be a soft convergent sequence of soft real numbers and converges $\tilde{l}$ and moreover if $\tilde{a}_{n} \widetilde{\leq} \bar{a} \forall n \in N$

Then $\tilde{\tilde{l}} \widetilde{\Xi}$.

Proof: since $<\tilde{\tilde{a}}_{n}>$ converges $\tilde{\tilde{l}}$ so according to definition $\lim _{n \rightarrow \infty} \tilde{\tilde{a}}_{n}=\tilde{l}$. We prove this result by contradiction let $\tilde{l} \widetilde{>} \bar{a}$ this implies that $\tilde{\tilde{l}}-\bar{a} \widetilde{\mp} \overline{0}$. Since $\lim \tilde{a}_{n}=\tilde{l}$. So then for every $\widetilde{\in} \widetilde{\leq} \overline{0}$ there exists soft integer $m$ such that $\left|\tilde{\tilde{a}}_{n}-\tilde{l}\right| \widetilde{\widetilde{\kappa}} \widetilde{\in}$ for all $n \widetilde{\Sigma} m$...

Since the choice of $\widetilde{\tilde{\epsilon}}$ is arbitrary so let us choose in particular $\widetilde{\in}=\tilde{l}-\bar{a}$ the

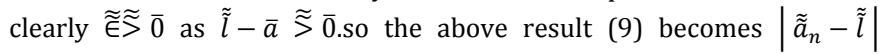
$\widetilde{\widetilde{\varepsilon}}=\tilde{l}-\bar{a}$, for all $n \widetilde{\geq} m$ this implies $\left|\tilde{a}_{n}-\tilde{l}\right| \widetilde{\widetilde{l}} \tilde{\tilde{l}}-\bar{a}$ for all $n \widetilde{\mathbb{\geq}} m$ implies that $-(\tilde{\tilde{l}}-\bar{a}) \widetilde{\widetilde{\alpha}} \tilde{\tilde{a}}_{n}-\tilde{\tilde{l}} \widetilde{<} \tilde{\tilde{l}}-\bar{a}$ for all $n \widetilde{\Xi} m$ implies that

$\bar{a} \widetilde{<} \tilde{\tilde{a}}_{n} \widetilde{<} \overline{2} \tilde{l} \forall n \widetilde{\geq} m$. This implies that $\bar{a} \widetilde{<} \widetilde{\tilde{a}}_{n}$ for all $n \widetilde{\geq} m$. Which is clearly a contradiction. Hence $\tilde{l} \widetilde{\underline{a}} \bar{a}$. This finishes the proof.

Theorem 7. Let $<\tilde{\tilde{a}}_{n}>$ and $<\tilde{\tilde{b}}_{n}>$ are soft convergent sequences of soft real numbers and if $\tilde{\tilde{a}}_{n} \widetilde{\widetilde{s}}_{n}$ forall $n \widetilde{\widetilde{\epsilon}} N$.Then $\lim _{n \rightarrow \infty}\left(\tilde{\tilde{a}}_{n}\right) \widetilde{\widetilde{\Xi}} \lim _{n \rightarrow \infty}\left(\tilde{\tilde{b}}_{n}\right)$

Proof: let $\lim _{n \rightarrow \infty} \tilde{\tilde{a}}_{n}=\widetilde{\widetilde{l}}_{1}, \lim _{n \rightarrow \infty} \tilde{\tilde{b}}_{n}=\tilde{\bar{l}_{2}}$

We have to prove $\widetilde{\widetilde{l_{1}}} \widetilde{\widetilde{l_{2}}} \widetilde{\widetilde{l}_{2}}$

Suppose $\widetilde{l_{1}} \widetilde{\widetilde{l_{2}}}$ implies that $\widetilde{l_{1}}$

$-\widetilde{l_{2}} \widetilde{>} \overline{0}$ implies $\frac{\text { that }\left(\widetilde{l_{1}}-\widetilde{l_{2}}\right)}{2 \widetilde{>} \overline{0}}$. Since $\lim _{n \rightarrow \infty} \tilde{\tilde{a}}_{n}$

$=\widetilde{l_{1}}$ so according to definition for $\widetilde{\in} \widetilde{\bar{S}} \overline{0}$ there exists a natural number

$m_{1}$ such that $\mid \tilde{\tilde{a}}_{n}$

$-\widetilde{\widetilde{l}} \mid \widetilde{<} \widetilde{\in}$ for all $n \widetilde{\Sigma} m_{1}$ from here we can conclude that $\widetilde{l_{1}}$

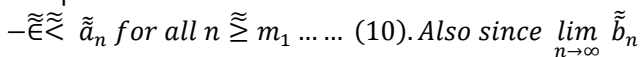

$=\widetilde{l_{2}}$ so according to definition for $\widetilde{\in} \widetilde{\bar{S}} \overline{0}$ there exists a natural number

$m_{1}$ such that $\mid \tilde{\tilde{b}}_{n}$

$-\widetilde{l_{2}} \mid \widetilde{\widetilde{\varepsilon}} \widetilde{\widetilde{E}}$ for all $n \widetilde{\widetilde{\geq}} m_{2}$ from here we can conclude that $\tilde{\tilde{b}}_{n} \approx \widetilde{\tilde{l}_{2}}$

$+\widetilde{\widetilde{\epsilon}}$ for all $n \widetilde{\Xi} m_{2}$

Let $\max \left(m_{1}, m_{2}\right)$

$=m$.then (10) and (11) become that $\tilde{\widetilde{l}}_{1}$

- $\widetilde{\widetilde{\varepsilon}} \widetilde{\tilde{a}_{n}}$ for all $n \widetilde{\Xi} m \ldots \ldots$ (10) and $\tilde{\tilde{b}}_{n} \widetilde{<} \widetilde{\tilde{l}_{2}}$

$+\widetilde{\widetilde{\epsilon}}$ for all $n \widetilde{\mathbb{\geq}} m$.......(11). Since $\left(\widetilde{\bar{l}_{1}}-\widetilde{\bar{l}_{2}}\right) / 2 \widetilde{>} \overline{0}$. Let $\widetilde{\widetilde{\epsilon}}$

$=\left(\widetilde{l_{1}}-\widetilde{l_{2}}\right) / 2$ implies that $2 \widetilde{\tilde{\epsilon}}=\left(\widetilde{\tilde{l}_{1}}-\widetilde{l_{2}}\right)$. Implies that $\widetilde{\widetilde{l}_{2}}$

$=\left(\widetilde{l_{1}}-2 \widetilde{\epsilon}\right)$. Then result (11) becomes $\tilde{\tilde{b}}_{n} \widetilde{\widetilde{<}} \widetilde{\bar{l}_{2}}$

- $\widetilde{\widetilde{\epsilon}}$ for all $n \widetilde{\geq} m$. But result (12) tells $\widetilde{l_{1}}$

- $\widetilde{\widetilde{\epsilon}} \widetilde{\tilde{a}_{n}}$ for all $n \widetilde{\leq} m$. This means that $\tilde{\tilde{b}}_{n} \widetilde{<} \tilde{\tilde{a}}_{n}$. But by hypothesis $\tilde{\tilde{b}}_{n} \widetilde{\widetilde{\leq}} \tilde{\tilde{a}}_{n}$. Hence $\tilde{\tilde{l}}_{1} \widetilde{\widetilde{\underline{\tau}}} \tilde{\tilde{l}}_{2}$.

\section{SUB SEQUENCES}

Definition 15: Suppose $\left\langle{\tilde{a^{\sim}}}_{n}\right\rangle$ be a soft sequence and $\left\langle\tilde{n}_{k}^{\sim}\right\rangle$ be a strictly soft increasing sequence in $\widetilde{N}$ that is

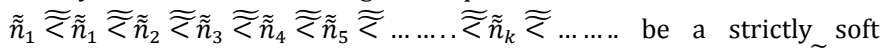
increasing sequence of soft natural numbers. Then the sequence $<\widetilde{\boldsymbol{a}}_{n_{k}}>$ is called a soft sub sequence of $<\tilde{\tilde{a}}_{n}>$.

Theorem 8. if the sequence $<\tilde{\tilde{a}}_{n}>$ of soft real numbers converges to soft limit $\tilde{l}$ if and only if every soft subsequence $<\tilde{\tilde{a}}_{n_{i}}>$ of the soft sequence $<\tilde{\tilde{a}}_{n}>$ also converges to soft limit $\tilde{l}$.

Proof: Since converges to $\tilde{l}$ then for every $\widetilde{\in} \widetilde{\bar{S}} \overline{0}$ there exists soft positive integer $\widetilde{\widetilde{M}}$ such that

$n \widetilde{\widetilde{M}} \approx\left|\tilde{\tilde{a}}_{n}-\tilde{l}\right| \widetilde{\widetilde{\varepsilon}} \widetilde{\epsilon}$

$\tilde{\tilde{l}}-\widetilde{\tilde{\epsilon}} \widetilde{\widetilde{a}} \tilde{\tilde{a}}_{n} \widetilde{<} \tilde{l}+\widetilde{\widetilde{\epsilon}}$ Since every term of $<\tilde{a}_{n_{i}}>$ is a term of $<\tilde{a}_{n}>$, so each term of $<\tilde{\tilde{a}}_{n}>$ from after $<\tilde{\tilde{a}}_{n}>m^{\text {th }}$ satisfies that $n_{i} \geq \widetilde{\widetilde{M}} \Rightarrow\left|\tilde{\tilde{a}}_{n_{i}}-\tilde{l}\right|<\widetilde{\widetilde{E}}$ $\tilde{l}$ is soft limit of $<\tilde{\tilde{a}}_{n_{i}}>$.

Hence $\lim _{n \rightarrow \infty} \tilde{\tilde{a}}_{n_{i}}=\tilde{\tilde{l}}$. Conversely, let $<\tilde{\tilde{a}}_{n_{i}}>$ converges to $\tilde{\tilde{l}}$. Then for every $\widetilde{\widetilde{\epsilon}} \widetilde{\bar{S}} \overline{0}$ there exists a soft positive integer $\widetilde{N}$ such that $n_{i} \geq \widetilde{\widetilde{N}} \Rightarrow\left|\tilde{\tilde{a}}_{n_{i}}-\tilde{l}\right|<$ $\widetilde{\widetilde{\epsilon}}$. Let in particular for $n_{i}=\tilde{\tilde{n}}$, we get

$\left|\tilde{\tilde{a}}_{n}-\tilde{\tilde{l}}\right|<\widetilde{\widetilde{\epsilon}} n_{i} \geq \widetilde{\widetilde{N}}$. Which finishes the proof.

Theorem 9. Every soft Sequence of soft real numbers has a soft convergent sub sequence.

Proof: For the purpose of this proof, we call the $m^{\text {th }}$ term $\tilde{\tilde{a}}_{m}$ of the soft sequence $<\tilde{a}_{n}>$ a "peak" if $\tilde{\tilde{a}}_{m} \widetilde{\Xi} \tilde{\tilde{a}}_{n}$ for all $n \widetilde{\leq} m$. Now two chances are taking birth:

Chance (i) if $\left.<\tilde{\tilde{a}}_{n}\right\rangle$ has infinitely many peak, order them by soft

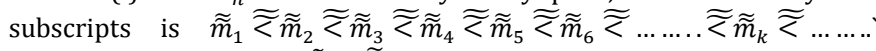
Since each of $\tilde{a}_{m_{1}}, \widetilde{\widetilde{a}}_{m_{2}} \ldots$ is a peak, we

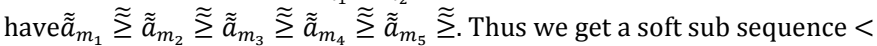
$\tilde{\tilde{a}}_{m_{k}}>$ of $<\tilde{\tilde{a}}_{n}>$ which is soft monotone decreasing. Also $<\tilde{\tilde{a}}_{m_{k}}>$ is soft bounded. Therefore, by soft monotone convergence theorem $<\tilde{\tilde{a}}_{m_{k}}>$ is soft convergent.

Chance (ii) let $<\tilde{\tilde{a}}_{n}>$ has a finite number of peaks, namely, $\tilde{\tilde{a}}_{m_{1}}, \widetilde{\widetilde{a}}_{m_{2}}, \ldots . . \widetilde{a}_{m_{r}}$. Put $k_{1}=m_{k}+1$, then $\widetilde{\widetilde{a}}_{k_{1}}$ is not a peak. Therefore, there exists a $k_{1} \widetilde{\Sigma} k_{2}$ such that $\tilde{\tilde{a}}_{k_{3}} \widetilde{\geq} \widetilde{\tilde{a}}_{k_{2}}$. Proceeding in a similar fashion, we get a soft sub sequence $<\tilde{\tilde{a}}_{k_{i}}>$ of $<\tilde{\tilde{a}}_{n}>$ which is soft monotone increasing. Also $<\tilde{\tilde{a}}_{n}>$ is soft bounded. Therefore, by soft monotone convergence criterion $<\tilde{\tilde{a}}_{k_{i}}>$ is soft convergent.

Definition16: The soft sequence $\left\langle{\tilde{a^{\sim}}}_{n}\right\rangle$ is bounded if and only if there exists soft number $\widetilde{\widetilde{M}} \widetilde{\widetilde{\epsilon}} \tilde{\tilde{R}}$ such that $\left|\tilde{\tilde{a}}_{n}\right| \widetilde{\widetilde{\sim}} \widetilde{\widetilde{M}}$ for all $n \in \widetilde{\widetilde{N}}$.

Theorem 10. if the sequence $<\tilde{\tilde{a}}_{n}>$ of soft real number is convergent then it is bounded.

Proof: Let $<\tilde{\tilde{a}}_{n}>$ converges to $\tilde{l}$ then for $\widetilde{\in} \widetilde{\mathrm{S}} \overline{1}$, there exists $m \in N$ such that

$\left|\tilde{\tilde{a}}_{n}-\tilde{\tilde{l}}\right|<\overline{1} \forall n \widetilde{\widetilde{s}} m$.

$\left|\tilde{\tilde{a}}_{n}\right|=\left|\tilde{\tilde{l}}+\tilde{\tilde{a}}_{n}-\tilde{\tilde{l}}\right|$

$$
\begin{aligned}
& \widetilde{\widetilde{<}}|\tilde{l}|+\left|\tilde{\tilde{a}}_{n}-\tilde{l}\right| \\
& \widetilde{\boldsymbol{z}} \tilde{\tilde{l}}+\overline{1}
\end{aligned}
$$

If we set $\widetilde{\widetilde{M}}=\operatorname{Max}\left\{\left|\tilde{\tilde{a}}_{1}\right|,\left|\tilde{\tilde{a}}_{2}\right|, \ldots\left|\tilde{\tilde{a}}_{n-1}\right|\right\}$,

Then $\left|\tilde{a}_{n}\right| \widetilde{<} \widetilde{\bar{M}}+\tilde{l}+\tilde{1}$ for all $n \in N$.

This shows that the soft sequence $<\tilde{\tilde{a}}_{n}>$ is bounded.

Theorem 11. if $<\tilde{x}_{n}>$ be a soft sequence bounded and if $\lim _{n \rightarrow \infty}\left(\tilde{\tilde{x}}_{n}\right)=\tilde{l}$. Then

$\lim _{n \rightarrow \infty} \tilde{\tilde{x}}_{n}^{2}=\tilde{\tilde{l}}^{2}$

Proof: given $\lim _{n \rightarrow \infty}\left(\tilde{x}_{n}\right)=\tilde{l}$. So according to definition for soft real number $\widetilde{\tilde{S}} \widetilde{0}$, there exists a soft natural number $\widetilde{\widetilde{m}}$ such that $\mid \tilde{\tilde{x}}_{n}-$ $\tilde{l} \mid \widetilde{\widetilde{\kappa}} \frac{\widetilde{\tilde{E}}}{\widetilde{\widetilde{M}+}|\tilde{l}|}, n \widetilde{\Xi} \widetilde{m} \ldots . . . .(\mathrm{i})$

Since $<\tilde{\tilde{a}}_{n}>$ is bounded. Therefore $\left|\tilde{\tilde{x}}_{n}\right| \widetilde{<} \widetilde{\widetilde{M}}, n \widetilde{\in} N$.......(ii). Consider $\left|\tilde{\tilde{x}}_{n}{ }^{2}-\tilde{\tilde{l}}^{2}\right|=\left|\left(\tilde{\tilde{x}}_{n}-\tilde{\tilde{l}}\right)\left(\tilde{\tilde{x}}_{n}-\tilde{\tilde{l}}\right)\right| \ldots \ldots \ldots . .($ iiii $)$. Since, $\left|\tilde{\tilde{x}}_{n}+\tilde{\tilde{l}}\right| \widetilde{\widetilde{\leq}}\left|\tilde{\tilde{x}}_{n}\right|+|\tilde{\tilde{l}}|$ implies that $\left|\tilde{\tilde{x}}_{n}+\tilde{l}\right| \widetilde{\underline{\underline{I}}} \widetilde{\widetilde{M}}+|\tilde{\tilde{l}}|, n \widetilde{\widetilde{\epsilon}} N$.........(iiii). Now, (iii) becomes

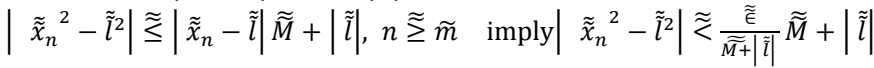
$, n \widetilde{\geq} \widetilde{m} .\left|\tilde{x}_{n}{ }^{2}-\tilde{l}^{2}\right| \widetilde{<} \widetilde{\epsilon}, n \widetilde{\geq} \widetilde{m} . \lim _{n \rightarrow \infty} \tilde{\tilde{x}}_{n}{ }^{2}=\tilde{l}^{2}$. This completes the proof.

Theorem 12. if $<\tilde{x}_{n}>$ be a soft sequence of soft real numbers that converges to soft limit $\tilde{l}$ and suppose that $\tilde{x}_{n} \widetilde{\geq} \overline{0}$. Then the soft sequence $\sqrt{\left(\tilde{\tilde{x}}_{n}\right)}$ of positive square roots converges and $\lim _{n \rightarrow \infty} \sqrt{\left(\tilde{\tilde{x}}_{n}\right)}=\sqrt{\tilde{\tilde{l}}}$.

Proof: Clearly $\tilde{\tilde{l}} \widetilde{\mathbb{}} \overline{0}$. This means that the assertion does make sense. So two chances are there. That is $\tilde{l} \widetilde{>} \overline{0}$ and $\tilde{\tilde{l}} \cong \overline{0}$. If $\tilde{\tilde{l}} \widetilde{\lessgtr} \overline{0}$ then $\sqrt{\tilde{l}} \widetilde{>} \overline{0}$.

Consider 
$\sqrt{\tilde{l}} \frac{\left(\sqrt{\left(\tilde{x}_{n}\right)}+\sqrt{\tilde{l}}\right)}{\sqrt{\left(\tilde{x}_{n}\right)}+\sqrt{\tilde{l}}} \mid=\frac{\left|\tilde{\tilde{x}}_{n}-\tilde{\tilde{l}}\right|}{\left|\sqrt{\left(\tilde{\tilde{x}}_{n}\right)}+\sqrt{\tilde{l}}\right|} \widetilde{\widetilde{\underline{x}}} \frac{\left|\tilde{x}_{n}-\tilde{l}\right|}{\sqrt{\tilde{l}}}$ for all $n \widetilde{\widetilde{z}} \widetilde{m}$

$\left.\lim _{n \rightarrow \infty} \mid \sqrt{\left(\tilde{\tilde{x}}_{n}\right)}-\sqrt{\tilde{\tilde{l}}}\right) \mid \widetilde{\widetilde{\Sigma}} \overline{0} . \quad$ But $\left.\lim _{n \rightarrow \infty} \mid \sqrt{\left(\tilde{\tilde{x}}_{n}\right)}-\sqrt{\tilde{\tilde{l}}}\right) \mid \widetilde{\widetilde{x}} \overline{0}$. This implies that $\left.\lim _{n \rightarrow \infty} \mid \sqrt{\left(\tilde{\tilde{x}}_{n}\right)}-\sqrt{\tilde{\tilde{l}}}\right) \mid \cong \overline{0}$. This implies $\lim _{n \rightarrow \infty} \sqrt{\left(\tilde{\tilde{x}}_{n}\right)}=\sqrt{\tilde{\tilde{l}}}$. In a similar fashion we can easily prove the second case.

\section{SOFT MONOTONE SEQUENCES OR SOFT MONOTONE SEQUENCES AND THEIR CONVERGENCE.}

The monotone convergences theorems establish the existence of the limit of a bounded monotone soft sequence. It also provides us a fashion of calculating the limit of the soft sequences provided we can evaluate the supremum or infimum. Sometimes it is difficult this supremum or infimum. But once we know by one way or the other that it exists, it is often possible to evaluate the limit by other techniques. In this portion we will also discuss soft monotone increasing un-bounded and soft monotone decreasing un-bounded soft sequences and we will prove that they diverge to plus infinity and minus infinity respectively.

Definition 17: let $<{\tilde{a^{\sim}}}_{n}>$ be a soft sequence of soft real numbers then $\tilde{\tilde{a}}_{n}>$ is said to be monotonic increasing or monotonically (or nondecreasing) if $\tilde{\tilde{a}}_{n} \underset{\widetilde{\leq}}{\widetilde{a_{n}}} \tilde{a}_{n+1} \forall n \widetilde{\widetilde{\epsilon}} N$. if $\tilde{\tilde{a}}_{n} \widetilde{<} \tilde{\tilde{a}}_{n+1}, \forall n \widetilde{\widetilde{\epsilon}} N$.Then it is said to be strictly monotonic increasing. A soft monotonic increasing sequence is always bounded below

Definition 18: let $\left\langle a_{n}^{\sim}>\right.$ be a soft sequence of soft real numbers then $\tilde{\tilde{a}}_{n}>$ is said to be monotonic increasing or monotonically (or nonincreasing) if $\tilde{\tilde{a}}_{n} \widetilde{\widetilde{z}} \tilde{\tilde{a}}_{n+1} \forall n \widetilde{\widetilde{\epsilon}} N$. if $\tilde{\tilde{a}}_{n} \widetilde{\widetilde{s}} \widetilde{\tilde{a}}_{n+1}, \forall n \widetilde{\widetilde{\epsilon}} N$. Then it is said to be strictly monotonic decreasing. A soft sequence $<\tilde{\tilde{a}}_{n}>$ is said to monotone or monotonic if it is either monotonic increasing or monotonic decreasing. A soft monotonic decreasing sequence is always bounded above.

Theorem 13. Every soft monotonic increasing sequence which is bounded above tends to its soft least upper bound

Proof: let $<\tilde{\tilde{a}}_{n}>$ be a soft monotonic increasing sequence having $M$ as its l.u.b. Then $\tilde{\tilde{a}}_{n} \widetilde{\widetilde{\leq}} M$ for all $n$. Now for $\widetilde{\widetilde{\epsilon}} \widetilde{\bar{S}} \overline{0}, M-\widetilde{\widetilde{\epsilon}} \widetilde{<} \tilde{\tilde{a}}_{n}$ for at least one value of $n$. Let us suppose that particular value be $n=N$. $M-\widetilde{\tilde{\epsilon}} \widetilde{<} \tilde{\tilde{a}}_{N}$. Since $<$ $\tilde{\tilde{a}}_{n}>$ is soft monotonically increasing sequence. So we have the following picture $\tilde{a}_{n} \widetilde{\widetilde{\leq}} \tilde{\tilde{a}}_{N+1} \widetilde{\widetilde{\leq}} \widetilde{\tilde{a}}_{N+2} \widetilde{\widetilde{\leq}}, \ldots .$. . Providing that $M-\widetilde{\widetilde{\epsilon}} \widetilde{<} \tilde{a}_{N} \quad \forall n \widetilde{\Xi} N \ldots .$. (i). Also $\tilde{\tilde{a}}_{n} \widetilde{\widetilde{\leq}} M$ for all value of $\mathrm{n}$. this implies $\tilde{\tilde{a}}_{n} \widetilde{\widetilde{\leq}} M+\widetilde{\widetilde{\epsilon}}$ for all value of n..... (ii). Mixing (i) and (ii) we have $\left|\tilde{\tilde{a}}_{n}-M\right| \widetilde{\widetilde{<}} \widetilde{\tilde{\epsilon}}$ for all $n \widetilde{\widetilde{\geq}} N$. Showing that $<\tilde{a}_{n}>$ converges to $M$

Theorem 14. Every soft monotonic decreasing sequence which is bounded below tends to its greatest lower bound

Proof: let $<\tilde{\tilde{a}}_{n}>$ be a soft monotonic decreasing sequence having $m$ as its l.u.b. Then $\tilde{\tilde{a}}_{n} \widetilde{\widetilde{\leq}} m$ for all $n$. Now for $\widetilde{\widetilde{E}} \widetilde{\bar{S}} \overline{0}, m+\widetilde{\widetilde{E}} \widetilde{\underline{\Xi}} \tilde{a}_{n}$ for at least one value of $n$. Let us suppose that particular value ben $=N$. $M+\widetilde{\widetilde{E}} \widetilde{\underline{\Xi}} \tilde{\tilde{a}}_{N}$. Since $<$ $\tilde{\tilde{a}}_{n}>$ is soft monotonically decreasing sequence. So we have the following

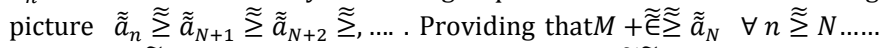
(i). Also $m \widetilde{\leq} \tilde{a}_{n}$ for all value of $n$. this implies $M-\widetilde{\widetilde{\epsilon}} \widetilde{\leq} \tilde{a}_{n}$ for all value of n..... (ii). Mixing (i) and (ii) we have $\left|\tilde{\tilde{a}}_{n}-m\right| \widetilde{<} \widetilde{\tilde{\epsilon}}$ for all $n \widetilde{\Xi} N$. Showing that $<\tilde{\tilde{a}}_{n}>$ converges to $m$.

Theorem 15. Every soft monotonic increasing sequence which is unbounded above tends to plus infinity.

Proof: let $<\tilde{\tilde{a}}_{n}>$ be a soft monotonic increasing sequence. So we have the following picture $\tilde{a}_{n} \widetilde{\widetilde{\leq}} \tilde{\tilde{a}}_{N+1} \widetilde{\widetilde{\leq}} \tilde{\tilde{a}}_{N+2} \widetilde{\widetilde{\leq}}, \ldots .$. . Providing that $M-\widetilde{\widetilde{\epsilon}} \widetilde{<} \tilde{a}_{N}$ $\forall n \widetilde{\geq} N$...... (i). since $<\tilde{a}_{n}>$ is not bounded above. So according to definition for any soft real number $\tilde{\tilde{G}}$ (how-ever large we can search out a soft natural number $\underset{\widetilde{m}}{\widetilde{\tilde{\epsilon}}} N$ such that $\tilde{\tilde{a}}_{m} \widetilde{\widetilde{\sigma}} \tilde{\tilde{G}}$...... (ii). Mixing (i) and (ii), we have $G \widetilde{<} \widetilde{\tilde{a}}_{m} \widetilde{\widetilde{\leq}} \tilde{\tilde{a}}_{m+2} \widetilde{\widetilde{\leq}}, \ldots .$. This implies that $G \widetilde{<} \widetilde{\tilde{a}}_{n} \quad$ for all $n \widetilde{\leq} m$ or $\tilde{\tilde{a}}_{n} \widetilde{>} \tilde{\tilde{G}} n \widetilde{\widetilde{\Sigma}} m$.This means that $<\tilde{\tilde{a}}_{n}>$ diverges to infinity. This finishes the proof.

Theorem 16. Every soft monotonic decreasing sequence which is unbounded below tends to minus infinity.

Proof: let $<\tilde{a}_{n}>$ be a soft monotonic decreasing sequence. So we have the following picture $\tilde{\tilde{a}}_{m} \widetilde{\underline{\leq}} \tilde{\tilde{a}}_{m+1} \widetilde{\underline{\Sigma}} \tilde{\tilde{a}}_{m+2} \widetilde{\underline{\leq}}, \ldots$. . (iii). Since $<\tilde{\tilde{a}}_{n}>$ is not bounded below. So for any soft number however small we can find a soft natural number $\widetilde{\widetilde{m}} \widetilde{\epsilon} N$ Such that $\tilde{\tilde{a}}_{n} \widetilde{<} \widetilde{K}$....(iiii). Mixing (iii) and (iiii) we have $\widetilde{K} \widetilde{\tilde{a}_{m}} \widetilde{\widetilde{z}} \tilde{\tilde{a}}_{m+1} \widetilde{\geq} \tilde{\tilde{a}}_{m+2} \widetilde{\widetilde{\geq}}, \ldots$.

$\widetilde{K} \widetilde{>} \tilde{a}_{n} n \widetilde{\Sigma} m$. This means that $<\tilde{a}_{n}>$ diverges to minus infinity.

\section{SOFT SQUEEZE THEOREM}

The next result asserts that if a soft sequence is "Squeezed "or "Pinched" between two other soft sequences that converges to the same soft limit then it must also Converges to the same soft limit.

Theorem 17. if $<\tilde{a}_{n}>,<\tilde{\tilde{b}}_{n}>$ and $<\tilde{\tilde{c}}_{n}>$ soft sequences such that $\tilde{\tilde{a}}_{n} \widetilde{\widetilde{\underline{b}}} \tilde{\tilde{b}}_{n} \widetilde{\widetilde{\underline{\tau}}} \tilde{\tilde{c}}_{n}$ for all $n \widetilde{\widetilde{\epsilon}} N$, and if $\lim _{n \rightarrow \infty} \tilde{\tilde{a}}_{n}=\lim _{n \rightarrow \infty} \tilde{\tilde{c}}_{n}=\tilde{\tilde{l}}$, then $\lim _{n \rightarrow \infty} \tilde{\tilde{b}}_{n}=\tilde{l}$. Proof: since $\lim _{n \rightarrow \infty} \tilde{\tilde{a}}_{n}=\tilde{l}$ and $\lim _{n \rightarrow \infty} \tilde{\tilde{c}}_{n}=\tilde{n}$, then for $\widetilde{\in} \widetilde{\bar{S}} \overline{0}$ since converges to $\tilde{l}$ there exists soft positive integer $n_{1}, n_{1} \widetilde{\widetilde{\epsilon}} N$ such that $n \widetilde{\mathbb{\geq}} n_{1} \Rightarrow\left|\tilde{\tilde{a}}_{n}-\tilde{l}\right| \widetilde{\widetilde{\varepsilon}} \widetilde{\tilde{\epsilon}}$ and $n \widetilde{\mathbb{z}} n_{2} \Rightarrow\left|\tilde{\tilde{c}}_{n}-\tilde{\tilde{l}}\right| \widetilde{\widetilde{\varepsilon}} \widetilde{\widetilde{\epsilon}}$. Let $M=\max \left(n_{1}, n_{1}\right)$. Then $n \widetilde{\geq} M$ implies $\left|\tilde{a}_{n}-\tilde{l}\right| \widetilde{<} \widetilde{\epsilon}$ implies $\tilde{l}-\widetilde{\epsilon} \widetilde{<} \tilde{a}_{n} \widetilde{<} \tilde{l}+\widetilde{\epsilon}$ Then $n \widetilde{\Xi} M$

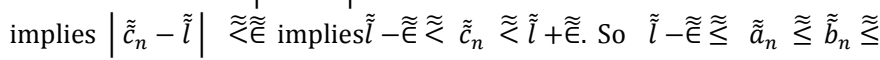

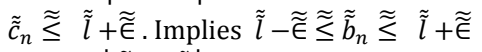
Implies $\left|\tilde{b}_{n}-\tilde{\tilde{l}}\right| \widetilde{\widetilde{<}} \widetilde{\in}$ for all $n \widetilde{\widetilde{S}} N$ or $\lim _{n \rightarrow \infty} \tilde{\tilde{b}}_{n}=\tilde{\tilde{l}}$.

\section{SOFT CAUCHY SEQUENCE}

Definition 19: A soft sequence $\left\langle x_{n}^{\sim}>\right.$ is said to be a soft Cauchy Sequence if for every $\in^{\sim} \mathcal{S}^{\sim} \mathcal{\sim}$, there exists a positive soft integer $N \widetilde{s u c h}$ that $\left|\tilde{x_{n}}-\tilde{x_{m}}\right|$

$\widetilde{<} \widetilde{\in}$ for all $n, m \widetilde{\geq} N$. Thus if a soft sequence is a soft Cauchy sequence, then for every soft real number $\widetilde{\in} \widetilde{\bar{S}} \overline{0}$ there exists a positive soft integer $\widetilde{\widetilde{M}}$ such that the difference between any two soft elements which come from and after the $M t h$ term is less than $\widetilde{\in}$. This means that the soft elements of the soft sequence eventually get closer in this case which produces interesting situation. When such type of situation is born then it guarantees that the terms are very mixed to-gather which is click towards the convergence and boundedness.

\section{Theorem 18. Every Soft Cauchy sequence is bounded.}

Proof: Let $<\tilde{x}_{n}>$ be a soft Cauchy sequence. Then according to definition for every soft real number $\widetilde{\in} \widetilde{\bar{S}} \overline{0}$ there exists a positive integer $\mathrm{N}$ such that $\left|\tilde{\tilde{x}}_{n}-\tilde{\tilde{x}}_{m}\right| \widetilde{\widetilde{\varepsilon}} \widetilde{\widetilde{E}}$ for all $n, m \widetilde{\geq} N$.

In particular, for $m=N$, we have $\left|\tilde{x}_{n}-\tilde{x}_{N}\right| \widetilde{\widetilde{\varepsilon}} \widetilde{\widetilde{\epsilon}}$ for all $n, \widetilde{\Xi} N$. Implies

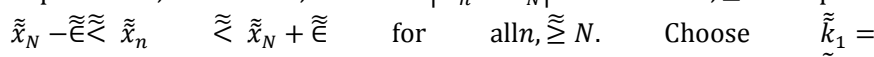
$\min \left\{\tilde{x}_{1}, \tilde{x}_{2}, \tilde{x}_{3}, \tilde{x}_{4}, \ldots . . \tilde{x}_{N-1}, \tilde{x}_{N-\tilde{\epsilon}}\right\} \quad$ and $\quad \tilde{k}_{2}=$ $\min \left\{\tilde{\tilde{x}}_{1}, \tilde{\tilde{x}}_{2}, \tilde{\tilde{x}}_{3}, \tilde{\tilde{x}}_{4}, \ldots . . \tilde{\tilde{x}}_{N-1}, \tilde{\tilde{x}}_{N+\widetilde{\epsilon}}\right\}$. Then $\tilde{\tilde{k}}_{1} \widetilde{\widetilde{\leq}} \tilde{\tilde{x}}_{n} \widetilde{\widetilde{\underline{x}}} \tilde{\tilde{k}}_{2}$ for all $n, \widetilde{\Xi} N$. Hence $<\tilde{x}_{n}>$ is a bounded soft sequence.

Theorem 19. Every soft convergent sequence is soft Cauchy sequence.

Proof: Let $\left\langle\tilde{\tilde{x}}_{n}\right\rangle$ be any soft convergent sequence and moreover converges to $\tilde{\tilde{x}}$. Then for every $\widetilde{\in} \widetilde{>} \overline{0}$ there exists a positive soft integer $\mathrm{N}$

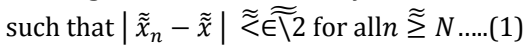

Let $m, \widetilde{\geq} N$ be any positive soft integer. Then (1) becomes $\left|\tilde{x}_{m}-\tilde{x}\right| \widetilde{\widetilde{\epsilon}} \widetilde{\mathbb{} 2}$ for all $m \widetilde{\Sigma} N$.....(2). Thus $\left|\tilde{x}_{n}-\tilde{\tilde{x}}_{m}\right|=\left|\tilde{x}_{n}-\tilde{\tilde{x}}+\tilde{\tilde{x}}-\tilde{x}_{m}\right| \widetilde{\underline{x}}\left|\tilde{\tilde{x}}_{n}-\tilde{\tilde{x}}\right|$

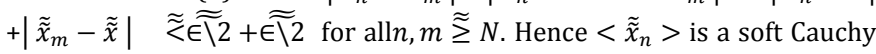
sequence.

Theorem 20. If $\lim _{n \rightarrow \infty} \tilde{S}_{n}=\tilde{S}$ and $\tilde{\tilde{x}}_{n}=\frac{1}{n}\left(\tilde{S}_{1}, \tilde{S}_{2}, \tilde{S}_{3}, \ldots \ldots \ldots \tilde{S}_{n}\right)$ where $n=$ $1,2,3 \ldots$. then $\lim _{n \rightarrow \infty} \tilde{\tilde{x}}_{n}=\tilde{\tilde{S}}$.

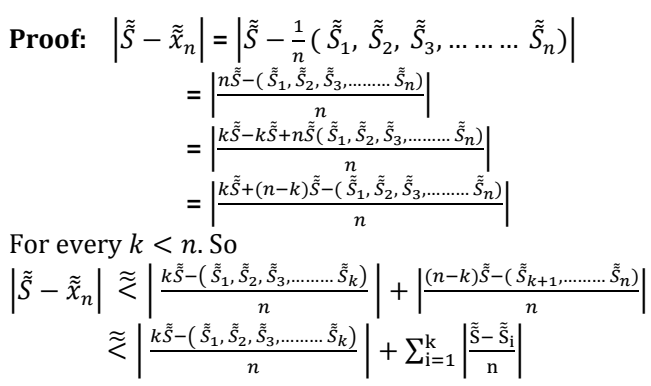


Let $\widetilde{\tilde{\epsilon}} \widetilde{\mathrm{S}} 0$. Choose $\mathrm{k}$ so large then $i>k \Rightarrow\left|\tilde{\tilde{S}}-\tilde{\mathrm{S}_{\mathrm{i}}}\right| \widetilde{\widetilde{\epsilon}} \widetilde{\widetilde{\epsilon}} / 2$

So for $n>k$,

$\left|\tilde{\tilde{S}}-\tilde{\tilde{x}}_{n}\right| \widetilde{\widetilde{<}}\left|\frac{k \tilde{\tilde{S}}-\left(\tilde{\tilde{S}}_{1}, \tilde{\tilde{S}}_{2}, \tilde{\tilde{S}}_{3}, \ldots \ldots \ldots \tilde{S}_{k}\right)}{n}\right|+\widetilde{\widetilde{E}} / 2$

But $\left|k \tilde{\tilde{S}}-\frac{1}{n}\left(\tilde{\tilde{S}}_{1}, \tilde{\tilde{S}}_{2}, \tilde{\tilde{S}}_{3}, \ldots \ldots \ldots \tilde{\tilde{S}}_{k}\right)\right|$ is constant so that the soft sequence $\left\{\left|\frac{k \tilde{S}-\left(\tilde{S}_{1}, \tilde{\tilde{S}}_{2}, \tilde{\tilde{S}}_{3}, \ldots \ldots \ldots \tilde{S}_{k}\right)}{n}\right|\right\} \rightarrow 0$ as $n \rightarrow \infty$. Hence there is an integer $N_{0}>k$ such that $n>N_{0} . \Rightarrow\left|\frac{k \tilde{\tilde{S}}-\left(\tilde{\tilde{S}}_{1}, \tilde{\tilde{S}}_{2}, \tilde{\tilde{S}}_{3}, \ldots \ldots \ldots \tilde{\tilde{S}}_{k}\right)}{n}\right| \widetilde{\widetilde{\kappa}} \widetilde{\widetilde{\epsilon}} / 2$.

Hence $\left|\tilde{\tilde{S}}-\tilde{x}_{n}\right| \widetilde{\widetilde{\varepsilon}} / 2+\widetilde{\tilde{\epsilon}} / 2=\widetilde{\epsilon}$

Therefore $\lim _{n \rightarrow \infty} \tilde{x}_{n}=\tilde{S}$.

Theorem 21. If $\left\{\tilde{a}_{n}\right\}$ be a soft sequence of positive terns, then $\lim _{n \rightarrow \infty}\left(\tilde{\tilde{a}}_{n}\right)^{\frac{1}{n}}=$ $\lim _{n \rightarrow \infty} \frac{\tilde{a}_{n+1}}{\tilde{a}_{n}}$, provided $\lim _{n \rightarrow \infty} \frac{\tilde{a}_{n+1}}{\tilde{a}_{n}}$ exists, whether finite or infinite.

Proof: $\lim _{n \rightarrow \infty} \frac{\tilde{a}_{n+1}}{\tilde{a}_{n}}=\tilde{l}($ finite $)$. Then for some real number $\widetilde{\tilde{E}} \widetilde{\mathrm{S}} 0$ there exist a positive integer m such that

$\left|\frac{\tilde{\tilde{a}}_{n+1}}{\tilde{\tilde{a}}_{n}}-\tilde{\tilde{l}}\right| \widetilde{\widetilde{\varepsilon}} \frac{\tilde{\tilde{a}}_{n+1}}{\tilde{a}_{n}} \widetilde{\approx} \tilde{l}+\widetilde{\tilde{\epsilon}} / 2$

For $n=m, m+1, m+2, \ldots m+p-1, p \in N$ we have

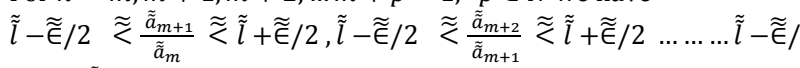

$2 \widetilde{\widetilde{\alpha}} \frac{\tilde{a}_{m+p}}{\tilde{a}_{m+p-1}} \widetilde{\widetilde{l}} \tilde{l}+\widetilde{\epsilon} / 2$

On multiplying these inequalities, we get

$(\tilde{l}-\widetilde{\epsilon} / 2)^{p} \widetilde{\widetilde{<}\left(\frac{\tilde{a}_{m+p}}{\tilde{a}_{m}}\right)} \widetilde{\widetilde{<}}(\tilde{\tilde{l}}+\widetilde{\epsilon} / 2)^{p} \ldots .(\tilde{l}-\widetilde{\epsilon} / 2)^{\frac{p}{m+p}} \widetilde{\widetilde{<}}\left(\frac{\tilde{\tilde{a}}_{m+p}}{\tilde{\tilde{a}}_{m}}\right)^{\frac{1}{m+p}} \widetilde{\widetilde{l}}(\tilde{l}+$ $\widetilde{\epsilon} / 2)^{\frac{p}{m+p}}$

$(\tilde{l}-\widetilde{\epsilon} / 2)^{\frac{p}{m+p}} \widetilde{<}\left(\tilde{\tilde{a}}_{m}\right)^{\frac{1}{m+p}} \widetilde{<}\left(\tilde{\tilde{a}}_{m+p}\right)^{\frac{1}{m+p}} \widetilde{<}(\tilde{l}+\widetilde{\widetilde{\epsilon}} / 2)^{\frac{p}{m+p}}\left(\tilde{\tilde{a}}_{m}\right)^{\frac{1}{m+p}}$

Since $p \rightarrow \infty, \frac{1}{m+p} \rightarrow 0, \frac{p}{m+p} \rightarrow 1$

and $(\tilde{l}-\widetilde{\tilde{\epsilon}} / 2)^{\frac{p}{m+p}}\left(\tilde{\tilde{a}}_{m}\right)^{\frac{1}{m+p}} \rightarrow \tilde{l}-\widetilde{\tilde{\epsilon}} / 2$

and $(\tilde{\tilde{l}}+\widetilde{\widetilde{\epsilon}} / 2)^{\frac{p}{m+p}}\left(\tilde{\tilde{a}}_{m}\right)^{\frac{1}{m+p}} \rightarrow \tilde{l}+\widetilde{\widetilde{\epsilon}} / 2$

Therefore from (2) we have

$(\tilde{l}-\widetilde{\tilde{\epsilon}} / 2)-\widetilde{\widetilde{\epsilon}} / 2 \quad \widetilde{\approx}(\tilde{l}-\widetilde{\tilde{\epsilon}} / 2)^{\frac{p}{m+p}}\left(\tilde{\tilde{a}}_{m}\right)^{\frac{1}{m+p}} \widetilde{\approx}(\tilde{l}-\widetilde{\widetilde{\epsilon}} / 2)+\widetilde{\widetilde{\epsilon}} / 2$

$\Rightarrow \tilde{l}-\widetilde{\tilde{\epsilon}} \widetilde{\gtrless}(\tilde{l}-\widetilde{\tilde{\epsilon}} / 2)^{\frac{p}{m+p}}\left(\tilde{\tilde{a}}_{m}\right)^{\frac{1}{m+p}} \widetilde{\gtrless} \tilde{l}$

And from (3) we have

$(\tilde{l}+\widetilde{\tilde{\epsilon}} / 2)-\widetilde{\widetilde{\epsilon}} / 2 \quad \widetilde{<}(\tilde{l}+\widetilde{\tilde{\epsilon}} / 2)^{\frac{p}{m+p}}\left(\tilde{\tilde{a}}_{m}\right)^{\frac{1}{m+p}} \widetilde{\gtrless}(\tilde{l}+\widetilde{\tilde{\epsilon}} / 2)+\widetilde{\widetilde{\epsilon}} / 2$

$\Rightarrow \tilde{l} \widetilde{<}(\tilde{l}+\widetilde{\tilde{\epsilon}} / 2)^{\frac{p}{m+p}}\left(\tilde{\tilde{a}}_{m}\right)^{\frac{1}{m+p}} \widetilde{\widetilde{l}} \tilde{l}+\widetilde{\tilde{\epsilon}}$

From (4) we have

$$
\begin{array}{r}
\tilde{l}-\widetilde{\epsilon} \widetilde{<}(\tilde{l}-\widetilde{\epsilon} / 2)^{\frac{p}{m+p}}\left(\widetilde{\tilde{a}}_{m}\right)^{\frac{1}{m+p}} \widetilde{<}\left(\tilde{a}_{m+p}\right)^{\frac{1}{m+p}} \widetilde{<}(\tilde{l} \\
+\widetilde{\widetilde{\epsilon}} / 2)^{\frac{p}{m+p}}\left(\tilde{\tilde{a}}_{m}\right)^{\frac{1}{m+p}} \widetilde{\approx} \tilde{l}+\widetilde{\epsilon}
\end{array}
$$

$\Rightarrow \tilde{\tilde{l}}-\widetilde{\widetilde{\epsilon}} \widetilde{\widetilde{<}}\left(\tilde{\tilde{a}}_{m+p}\right)^{\frac{1}{m+p}} \approx \widetilde{\widetilde{l}}+\widetilde{\epsilon}$

$\Rightarrow \tilde{l}-\widetilde{\epsilon} \widetilde{<}\left(\tilde{a}_{n}\right)^{\frac{1}{n}} \widetilde{<} \tilde{l}+\widetilde{\epsilon}$, for all $n \widetilde{>} m$

$\Rightarrow\left|\left(\tilde{\tilde{a}}_{n}\right)^{\frac{1}{n}}-\tilde{\tilde{l}}\right| \widetilde{\widetilde{\kappa} \widetilde{\epsilon}}$

$\Rightarrow \lim _{n \rightarrow \infty}\left(\tilde{\tilde{a}}_{n}\right)^{\frac{1}{n}}=\tilde{l}$

Case II: Let $\lim _{n \rightarrow \infty} \frac{\tilde{\tilde{a}}_{n+1}}{\tilde{a}_{n}}=\infty$. Then for some positive integer $\mathrm{k}$, however larger, then there exists a positive integer $\mathrm{m}$, such that $\frac{\tilde{a}_{n+1}}{\tilde{a}_{n}} \widetilde{>}(k+1)$, for all $n \widetilde{>} m$

For $n=m, m+1, m+2, \ldots \ldots m+p-1, p \in N$

Then $\frac{\tilde{\tilde{a}}_{m+1}}{\tilde{a}_{m}} \widetilde{\mathrm{S}} k+1, \frac{\tilde{\tilde{a}}_{m+2}}{\tilde{\tilde{a}}_{m+1}} \widetilde{\mathrm{S}} k+2, \ldots, \frac{\tilde{\tilde{a}}_{m+p}}{\tilde{\tilde{a}}_{m}} \widetilde{\mathrm{S}}_{k+1}$

Multiplying all these inequalities, we get

$\frac{\tilde{a}_{m+p}}{\tilde{a}_{m}} \widetilde{>}(k+1)^{p}$

$\Rightarrow\left(\frac{\tilde{\tilde{a}}_{m+p}}{\tilde{\tilde{a}}_{m}}\right)^{\frac{1}{m+p}} \widetilde{>}(k+1)^{\frac{p}{m+p}}$

$\Rightarrow\left(\tilde{\tilde{a}}_{m+p}\right)^{\frac{1}{m+p}} \widetilde{>}\left(\tilde{\tilde{a}}_{m}\right)^{\frac{1}{m+p}}(k+1)^{\frac{1}{m+p}}$

Now $\lim _{p \rightarrow \infty}\left(\tilde{\tilde{a}}_{m}\right)^{\frac{1}{m+p}}(k+1)^{\frac{1}{m+p}}=\left(\tilde{\tilde{a}}_{m}\right)^{0}(k+1)^{1}=k+1$

$\Rightarrow k+1-\widetilde{\widetilde{\epsilon}} \widetilde{<}\left(\tilde{a}_{m}\right)^{\frac{1}{m+p}}(k+1)^{\frac{p}{m+p}} \widetilde{<} k+1+\widetilde{\widetilde{\epsilon}}$

From (6) and (7) we get

$\left(\tilde{\tilde{a}}_{m+p}\right)^{\frac{1}{m+p}} \widetilde{\lessgtr}\left(\tilde{\tilde{a}}_{m}\right)^{\frac{1}{m+p}} \widetilde{\lessgtr}(k+1)^{\frac{p}{m+p}} \widetilde{\lessgtr} k+1-\widetilde{\tilde{\epsilon}}$

$\Rightarrow\left(\tilde{\tilde{a}}_{m+p}\right)^{\frac{1}{m+p}} \widetilde{>} k, \forall p \in N$
$\Rightarrow\left(\tilde{\tilde{a}}_{n}\right)^{\frac{1}{n}} \widetilde{>} k \forall n \widetilde{>} m$

$\Rightarrow \lim _{n \rightarrow \infty}\left(\tilde{\tilde{a}}_{n}\right)^{\frac{1}{n}} \rightarrow \infty$.

Theorem 22. Let $<\tilde{\tilde{a}}_{n}>$ be a soft sequence such that $\lim _{n \rightarrow \infty} \frac{\tilde{\tilde{a}}_{n+1}}{\tilde{a}_{n}}=\tilde{\tilde{l}}$ where $|\tilde{l}| \widetilde{<} \overline{1}$, then $\lim _{n \rightarrow \infty} \tilde{\tilde{a}}_{n}=\overline{0}$

Proof: since $\lim _{n \rightarrow \infty} \frac{\tilde{a}_{n+1}}{\tilde{a}_{n}}=\tilde{l}$ there for $\widetilde{\widetilde{E}} \widetilde{\bar{S}} \overline{0}$ provided that $|\tilde{l}|+\widetilde{\widetilde{\epsilon}} \widetilde{\widetilde{1}} \overline{1}$, there is a positive integer $m$ such that $n \widetilde{\widetilde{\Sigma}} m$ implies that $\left|\frac{\tilde{a}_{n+1}}{\tilde{a}_{n}}-\tilde{\tilde{l}}\right| \widetilde{<} \widetilde{\tilde{\epsilon}}$ 'implies that $\left|\lim _{n \rightarrow \infty} \frac{\tilde{a}_{n+1}}{\tilde{a}_{n}}\right| \widetilde{\gtrless}|\tilde{l}|+\widetilde{\widetilde{\epsilon}}=\mathrm{k}$ where $k \widetilde{<} \overline{1}$. Implies that $\left|\tilde{\tilde{a}}_{n+1}\right| \widetilde{\widetilde{<}} k\left|\tilde{\tilde{a}}_{n}\right|$. Now changing $n$ to $m, m+1, m+2, n-1$

We have $\left|\tilde{\tilde{a}}_{n}\right| \widetilde{\widetilde{<}} k\left|\tilde{\tilde{a}}_{n-1}\right| \widetilde{\widetilde{<}} k^{2}\left|\tilde{\tilde{a}}_{n-2}\right| \widetilde{<} k^{n-m}\left|\tilde{\tilde{a}}_{n}\right|$ this implies that $\left|\tilde{\tilde{a}}_{n}\right| \widetilde{<} \frac{k^{n}\left|\tilde{\tilde{a}}_{m}\right|}{k^{m}}$

Since $k^{n}$ runs towards zero, as n runs towards infinity. Since $k \widetilde{<} \overline{1}$. This implies that $\lim _{n \rightarrow \infty} \tilde{\tilde{a}}_{n}=\overline{0}$

Theorem 23. Let $<\tilde{\tilde{a}}_{n}>$ be a soft sequence such that $\tilde{\tilde{a}}_{n} \widetilde{\widetilde{ }} \overline{0}$ for all $n \in$ $\widetilde{\widetilde{N}} \lim _{n \rightarrow \infty} \frac{\tilde{a}_{n+1}}{\tilde{a}_{n}}=\tilde{l}$ where $\tilde{l} \widetilde{>} \overline{1}$, then $\lim _{n \rightarrow \infty} \tilde{\tilde{a}}_{n}=\bar{\infty}$.

Proof: $\quad$ since $\tilde{l}$ 空 $\overline{1}$ then $\widetilde{\in}$ can be chosen such that $\tilde{l}-\widetilde{\in} \widetilde{>} \overline{1}$. Since $\lim _{n \rightarrow \infty} \frac{\tilde{a}_{n+1}}{\tilde{a}_{n}}=\tilde{l}$, for $\tilde{E} \widetilde{>} \overline{0}$. There is a positive integer $m$ such that $n \widetilde{\mathbb{\geq}} m$ implies $\left|\frac{\tilde{a}_{n+1}}{\tilde{a}_{n}}-\tilde{l}\right| \widetilde{\widetilde{\varepsilon}} \widetilde{\widetilde{\epsilon}}$ implies $\tilde{l}-\widetilde{\widetilde{\epsilon}} \widetilde{\widetilde{\alpha}} \frac{\tilde{a}_{n+1}}{\tilde{a}_{n}} \widetilde{\widetilde{l}} \tilde{l}+\widetilde{\epsilon}$. Thus, for all $n \widetilde{\geq} m$ $\frac{\tilde{a}_{n+1}}{\tilde{a}_{n}} \widetilde{>} \tilde{l}-\widetilde{\tilde{\epsilon}}=k$, where $\mathrm{k} \widetilde{>} \overline{1}$. Now giving values to $n, n-1, n-2, \ldots . m$,

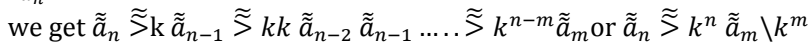

Now $\lim _{n \rightarrow \infty} \tilde{\tilde{a}}_{n}=\bar{\infty}$, since $k^{n}$ runs towards infinity as $n$ runs towards infinity became $k \widetilde{>} \overline{1}$.

Theorem 24. suppose $<\tilde{\tilde{a}}_{n}>\rightarrow \tilde{\tilde{a}}$ as $n \rightarrow \infty$. Then $e^{\tilde{\tilde{a}}_{n}} \rightarrow e^{a}$.

Proof: given $<\tilde{\tilde{a}}_{n}>\rightarrow \tilde{\tilde{a}}$ that is $\lim _{n \rightarrow \infty} \tilde{\tilde{a}}_{n}=\tilde{\tilde{a}}$ implies is $\lim _{n \rightarrow \infty} \tilde{\tilde{a}}_{n}-\tilde{\tilde{a}}=\overline{0}$. Let $\tilde{x}_{n}=\tilde{\tilde{a}}_{n}-\tilde{\tilde{a}}$. Then $\lim _{n \rightarrow \infty} \tilde{\tilde{x}}_{n}=\lim _{n \rightarrow \infty}\left(\tilde{\tilde{a}}_{n}-\tilde{\tilde{a}}\right)$. Implies $\lim _{n \rightarrow \infty} \tilde{\tilde{x}}_{n}=\overline{0}$. To prove the required result it is sufficient to prove that

$\lim _{n \rightarrow \infty} e^{\tilde{\tilde{x}}_{n}}=\overline{1}$. Let us choose $\overline{0}<\widetilde{\widetilde{\epsilon}} \widetilde{\widetilde{1}}$. Also let us choose $\widetilde{\widetilde{\epsilon}}_{1}=-\log (1-\widetilde{\widetilde{\epsilon}})$ and $\widetilde{\epsilon}_{2}=\log (1+\widetilde{\widetilde{\epsilon}})$. Then obviously $\widetilde{\widetilde{\epsilon}}_{1} \widetilde{\bar{S}} \overline{0}, \widetilde{\epsilon}_{2} \widetilde{\bar{S}} \overline{0}$. Now Min $\left\{\widetilde{\widetilde{\epsilon}}_{1}, \widetilde{\epsilon}_{2}\right\}$ $=\widetilde{\widetilde{E}}$.Then since $\lim _{n \rightarrow \infty} \tilde{\widetilde{x}}_{n}=\overline{0}$. So for the above $\widetilde{\widetilde{E}} \widetilde{\bar{S}} \overline{0}$, there exists a natural number $m$ such that $\left|\tilde{x}_{n}\right| \widetilde{<} \widetilde{\in}, n \widetilde{\Sigma} m$.

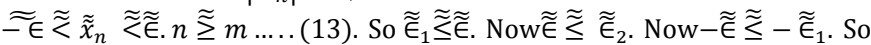
the above result becomes- $\widetilde{\epsilon}_{1} \widetilde{\varkappa}_{\tilde{x}_{n}} \widetilde{\widetilde{\kappa}} \widetilde{\epsilon}_{2} n \widetilde{\geq} m$. Using the chosen

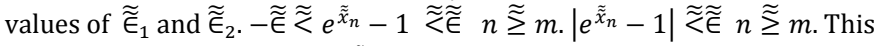
implies that as $n \rightarrow \infty$. thene $e^{\tilde{\tilde{a}}_{n}} \rightarrow e^{a}$. This finishes the proof.

Theorem 25. if $\tilde{\tilde{x}}_{n} \widetilde{>} \overline{0}$ for all $n \widetilde{\in} N$. And $\lim _{n \rightarrow \infty} \tilde{\tilde{x}}_{n}=\tilde{l}$ with $\tilde{l} \widetilde{\bar{\rho}} \overline{0}$. Then $\lim _{n \rightarrow \infty} \log \tilde{\tilde{x}}_{n}=\log \tilde{\tilde{l}}$.

Proof: $\quad$ Consider $\log \tilde{\tilde{x}}_{n}-\log \tilde{\tilde{l}}\left(\tilde{\tilde{x}}_{n}\right)=\log \left(\tilde{\tilde{x}}_{n}(\tilde{\tilde{l}})=\log \left(\tilde{\tilde{x}}_{n}+\tilde{l}-\tilde{l}\right) \backslash \tilde{\tilde{l}}\right.$ $=\log \left(\tilde{x}_{n}-\tilde{l}+\tilde{l}\right)\left(\tilde{l} l=\log \left(\tilde{x}_{n}-\tilde{l} / \tilde{l}+1\right)=\log \left(\tilde{x}_{n}+1\right) \quad\right.$ Where $\quad \tilde{x}_{n}=$

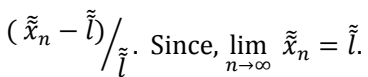

$\lim _{n \rightarrow \infty} \tilde{x}_{n}-\tilde{l}=\overline{0}$. Implies $\lim _{n \rightarrow \infty} \tilde{x}_{n}=\overline{0}$. To get the required result, we will prove that $\lim _{n \rightarrow \infty} \log \left(1+\tilde{x}_{n}\right)=\tilde{\tilde{l}}$ and for this let us choose soft positive number $\widetilde{\widetilde{E}} \widetilde{\bar{S}} \overline{0}$ and let us choose

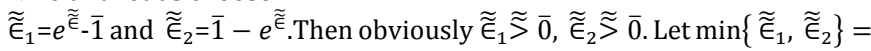
$\widetilde{\widetilde{\epsilon}}^{\prime}$

Since $\lim _{n \rightarrow \infty} \tilde{x}_{n}=\overline{0}$. So for $\widetilde{\widetilde{\epsilon}} / \widetilde{>} \overline{0}$.So we can find soft natural number $\widetilde{m}$ such that $\quad\left|\tilde{\tilde{x}}_{n}-\overline{0}\right| \widetilde{<} \widetilde{\epsilon}^{\prime}, n \widetilde{\Sigma} \widetilde{m}$. that $\quad\left|\tilde{\tilde{x}}_{n}\right| \widetilde{<} \widetilde{\widetilde{\epsilon}} / \quad, n \widetilde{\geq} \widetilde{m}$. $-\widetilde{\widetilde{\epsilon}} / \widetilde{\widetilde{<}} \tilde{\tilde{x}}_{n} \widetilde{\widetilde{<}} \widetilde{\widetilde{\epsilon}} /, n \widetilde{\mathbb{\geq}} m \ldots \ldots . . . \quad$ (A). $\quad$ since $\quad \min \left\{\widetilde{\widetilde{\epsilon}}_{1}, \widetilde{\widetilde{\epsilon}}_{2}\right\}=$

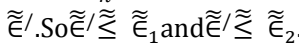

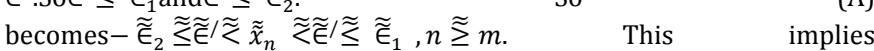
$-\widetilde{\widetilde{\epsilon}}_{2} \widetilde{<}_{\tilde{x}_{n}} \widetilde{<}_{\widetilde{\epsilon}_{1}}, n \widetilde{\geq} m$

$e^{\widetilde{\widetilde{\epsilon}}} \widetilde{<} \tilde{x}_{n}+\overline{1} \widetilde{<} e^{\widetilde{\widetilde{\epsilon}}}, n \widetilde{\geq} m$. Since, log is an increasing function. So,

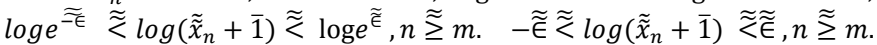
$\left|\log \left(\tilde{x}_{n}+\overline{1}\right)\right| \widetilde{<} \widetilde{\epsilon}, n \widetilde{\geq} m .\left|\log \left(\tilde{x}_{n}+\overline{1}\right)-\overline{0}.\right| \widetilde{<} \widetilde{\epsilon}, n \widetilde{\geq} m$. This implies 
that $\lim _{n \rightarrow \infty} \log \left(1+\tilde{\tilde{x}}_{n}\right)=\overline{0} \cdot \lim _{n \rightarrow \infty} \log \left(\tilde{\tilde{x}}_{n}\right)=\log \tilde{\tilde{l}}$. this completes the proof.

\section{CONCLUSION}

In this paper we have dealt with Soft Limit of Soft Sequences in a meticulously way which is foot path for the convergence of soft sequences. Some celebrated theorems related to soft convergence are lined up. Soft Sub Sequences are also reflected. Some theorems related to soft sub sequences are also discussed. Soft boundedness of soft Sequences is also discussed in a beautiful way. Soft Squeeze result is absorbed. Soft monotonicity of soft sequences is seen. Some related soft theorems are also inked. Soft Cauchy Sequences and their linkage with bounded sequences are also reflected. Soft Cauchy first and second theorems are also discussed.

I have fastidiously studied numerous homes on the behalf of soft Real Analysis, and lastly, I determined that soft Real Analysis is totally linked or in other sense we can correctly say that soft Real Analysis are connected with Soft structures. Provided if it is related with structures then it gives the idea of non-linearity beautifully. In other ways we can rightly say soft Real Analysis is somewhat directly proportional to non-linearity. Although we use non-linearity in Applied Math. So it is not wrong to say that soft Real Analysis is applied Math in itself. It means that soft Real Analysis has the taste of both of pure and applied math. In future I will discuss discussed results related with soft integral. We expect that these results in this article will do help the researchers for strengthening the tool box of soft Real Analysis structures. In the forthcoming, we spread the idea of soft Cauchy general principle of Convergence, Riemann Integral and related results and much more related to this particular area. The authors declare that there are no conflicts of interest regarding the Publication of this article.

\section{ACKNOWLEDGMENTS}

The authors are thankful to the reviewer(s) for valuable comments.

\section{REFERENCES}

[1] Zadeh, L.A. 1965. Fuzzy set. Infor Global Financial Controller, 8, 338-353.

[2] Molodtsov, D. 1999. Soft set theory-_rst results. Computers \& Mathematics with Applications, 37 (4/5), 19-31.

[3] Maji, P.K., Biswas, R., Roy, A.R. 2003. On soft set theory. Computers \& Mathematics with Applications, 45, 555- 562.

[4] Maji, P.K., Biswas, R., Roy, A.R. 2001. Fuzzy soft sets. The Journal of fuzzy Math., 9 (3), 589- 602.

[5] Shabir, M., Naz, M. 2011. On soft topological spaces. Computers \& Mathematics with Applications, 61, 1786-1799.

[6] Hac, A., Naim, C. 2007. Soft sets and soft groups. Information's Sciences, 177, 2726- 2735 .

[7] Nazmul, S.K., Samanta, S.K. 2010. Soft topological groups. Kochi Journal of Mathematics 5, 151-161.16.

[8] Das, S., Samanta, S.K. 2013. On Soft Metric Spaces. Journal of Fuzzy Mathematics, 21(3), 551-576.

[9] Das, S., Majumdar, P., Samanta, S.K. 2015. On soft linear spaces and soft normed linear Spaces. Annals of Fuzzy Mathematics and Informatics, 9(1), 91-109.

[10] Khattak, A.M. Soft Separation Axioms in Soft Single Point Spaces and In Soft Ordinary Spaces, Asian Journal of Mathematics and Computer Research, in press.

[11] Tantawy, O.A., Hassan,R.M. 2016. Soft Real Analysis. Journal of Progressive Research in Mathematics, 8(1), 1207-1219.

[12] Thakur, R., Samanta, S.K. 2015. Soft Banach Algebra. Annals of Fuzzy Mathematics and Informatics, 10(3), 397-412.

[13] Das, S., Samanta, S.K. 2012. Soft Real Sets, Soft Real Numbers and their properties. The Journal of Fuzzy Mathematics, 20(3), 551-576. 